\title{
PAPER \\ Adaptive Reversible Data Hiding via Integer-to-Integer Subband Transform and Adaptive Generalized Difference Expansion Method
}

\author{
Taichi YOSHIDA $^{\dagger \mathrm{a})}$, Student Member, Taizo SUZUKI ${ }^{\dagger \dagger \mathrm{b})}$, and Masaaki IKEHARA ${ }^{\dagger \mathrm{c})}$, Members
}

\begin{abstract}
SUMMARY We propose an adaptive reversible data hiding method with superior visual quality and capacity in which an adaptive generalized difference expansion (AGDE) method is applied to an integer-to-integer subband transform (I2I-ST). I2I-ST performs the reversible subband transform and the AGDE method is a state-of-the-art method of reversible data hiding. The results of experiments we performed objectively and perceptually show that the proposed method has better visual quality than conventional methods at the same embedding rate due to low variance in the frequency domain.

key words: adaptive reversible data hiding, adaptive generalized difference expansion, frequency domain, integer-to-integer subband transform
\end{abstract}

\section{Introduction}

With the ongoing development of computer and communication networks, many data hiding methods, which are techniques to embed information in signals, have been proposed for various purposes such as copyright protection, authentication, covert communication, and forensic tracking [1][19]. In typical data hiding for images, 'cover-image', 'messages', and 'stego-image' refer to 'original image', 'embedded information', and 'image with embedded information', respectively. Stego-images are evaluated in four aspects: 1) robustness, which is the resistance to modifications such as malicious attacks and image processing, 2) capacity, which is the maximum volume of messages under a given visual quality, 3) security, which indicates whether undesirable receivers illegally extract messages or not, and 4) visual quality, which is an objective, perceptual measure of the degradation of the stego-image compared with the cover-image and the invisibility of embedding.

Generally, data hiding methods are categorized as either watermarking or steganography. The former pursues a high robustness while sacrificing the capacity while the latter pursues a high capacity while sacrificing the robustness, i.e., they are in a trade-off relationship. Hence, watermarking is mainly utilized for copyright protection, authentication, and so on [1], [2], and steganography applica-

Manuscript received April 1, 2013.

Manuscript revised August 19, 2013

${ }^{\dagger}$ The authors are with the Department of Electronics and Electrical Engineering, Keio University, Yokohama-shi, 223-8522 Japan.

${ }^{\dagger \dagger}$ The author is with the Faculty of Engineering, Information and Systems, University of Tsukuba, Tsukuba-shi, 305-8573 Japan.

a)E-mail: yoshida@tkhm.elec.keio.ac.jp

b)E-mail: taizo@cs.tsukuba.ac.jp

c)E-mail: ikehara@tkhm.elec.keio.ac.jp

DOI: 10.1587/transfun.E97.A.384 tions are covert communication, forensic tracking, and so on [3]. Steganography is evaluated by its capacity, bit-per-pixel (bpp), at the same visual quality, or vice versa.

Reversible methods can completely restore not only messages but also the cover-image if the stego-image has not been modified. The reversibility of the cover-image is considerably important for military, medical, and artistic images. Some reversible data hiding methods have been proposed based on the histogram shifting (HS) technique [4][8]. These methods trade a quite limited embedding capacity for high visual quality. Recent works [7], [8] have used HS with image interpolation. HS embedding is applied for differences between original and interpolated pixels, and there are typically few distortions. These methods deliver excellent visual quality at a low capacity. However, if a high capacity is required, the visual quality is drastically damaged due to iterative embedding.

Some reversible data hiding methods in the frequency domain have also been proposed [9]-[11]. In [9], HS is applied for the quantized discrete cosine transform (DCT). In [10], least significant bits (LSBs) are adaptively replaced in the frequency domain obtained via an integer-to-integer wavelet transform. For the joint photographic experts group (JPEG) [11], [20] has proposed embedding messages in the quantized DCT coefficients according to the allocation and number of zero/non-zero coefficients. However, they cannot achieve a high capacity because they cannot be iteratively applied.

For the realization of high capacity reversible data hiding, the difference expansion (DE) method has been proposed [12]. This method divides images into two pixel pairs and then one pixel value is predicted from the other. A 1-bit message is embedded in the difference between the original and predicted pixels, called a stego-difference. The stegopixel is produced by adding the predicted pixel and stegodifference. This method embeds 1 bit into 2 pixels, i.e., its capacity is 0.5 bpp in one embedding process. In addition, it can be iteratively applied while the embedded pixel values have no-over/underflow.

This DE has been expanded into prediction error expansion (PEE) methods [13]-[15] and a generalized DE [16]-[19]. The PEE methods improve prediction and embedding algorithms while the generalized DE embeds arbitrary bits into arbitrary pixels. In [18], $n \log _{2} k\left(k=2^{m}\right.$, $m \in \mathbb{N})$ bits are embedded in $n+1(n \in \mathbb{N})$ pixels, where $k$ determines the capacity of stego-images. To improve the visual quality, [19] has proposed an adaptive generalized DE 


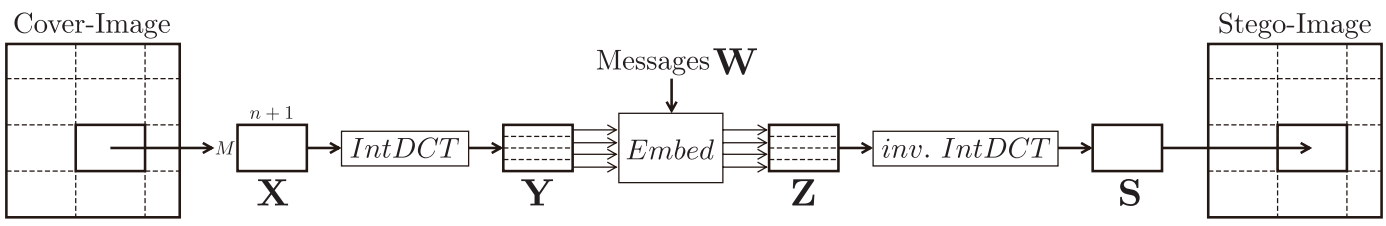

Fig. 1 Proposed data hiding algorithm. Notations are defined in Sect. 3.1 and channel $M$ is 4.

(AGDE) method that adaptively decides $k$ according to the variance of each cover-block. If the variance is high and $k$ is large, the embedding raises the massive distortions. The AGDE method efficiently distributes the distortions for better visual quality than the non-adaptive method [18], thus achieving both an excellent visual quality and a high capacity.

In this paper, we propose an adaptive reversible data hiding method with superior visual quality and capacity by applying the AGDE method [19] to the integer-to-integer subband transform (I2I-ST), which is briefly outlined in Fig. 1. Since natural images usually have a lower variance of each block of subbands in the frequency domain than one in the space domain, the distortions in the frequency domain are objectively lower at the same capacity. I2I-ST and its inverse transform are used for reversibility and to distribute the distortions to neighborhoods after embedding. Thus, the proposed method improves the visual quality. In this paper, we utilize the integer discrete cosine transform (IntDCT) [21]-[24], which is one of the most popular I2I-STs [25]-[27]. Experimental results objectively and perceptually show better visual quality than the conventional methods at the same embedding rate due to the low variance in the frequency domain.

The rest of this paper is organized as follows: Sect. 2 summarizes the DE based data hiding methods and the IntDCT. We then propose our adaptive reversible data hiding method in the frequency domain obtained via the I2I-ST and the AGDE in Sect. 3 and introduce its implementing structure in Sect. 4. Section 5 shows the results of several data hiding methods. We conclude in Sect. 6.

\section{Review}

\subsection{Generalized DE Method}

A high capacity reversible data hiding method has previously been proposed by Wang et al. [18]. Initially, the method divides a cover-image into several blocks, called cover-blocks, which contain $n+1$ pixels. Let $\mathbf{x} \in \mathbb{Z}^{n+1}$ be the cover-block and an element of $\mathbf{x}$ be its pixel. The method embeds $\mathbf{w} \in \mathbb{Z}^{n}$ in $\mathbf{x}$ to derive $\mathbf{y} \in \mathbb{Z}^{n+1}$, where $\mathbf{w}$ and $\mathbf{y}$ are messages and the stego-block, respectively. This embedding is defined as

$$
y_{j}=\left\{\begin{array}{ll}
k x_{j}-a_{n, k}(\mathbf{x}) & \text { for } j=0 \\
k x_{j}-a_{n, k}(\mathbf{x})+w_{j-1} & \text { for } j \neq 0
\end{array},\right.
$$

where $x_{j}, y_{j}$, and $w_{j}(j=0,1, \ldots, n)$ are elements of $\mathbf{x}, \mathbf{y}$, and $\mathbf{w}$, respectively, and $w_{j}$ should be represented by $\log _{2} k$ [bit] $\left(k=2^{m}, m \in \mathbb{N}\right) . k$ is a capacity parameter. $a_{n, k}(\mathbf{x})$ is defined as

$$
a_{n, k}(\mathbf{x})=\left\lfloor\frac{2(k-1) \sum_{j=0}^{n} x_{j}+n(k-1)}{2(n+1)}\right\rfloor,
$$

where $\lfloor\cdot\rfloor$ is the floor function. (1) means to embed $n \log _{2} k$ bits in $n+1$ signals, and $k$ controls the embedding rate.

In the extraction, from (1), $\mathbf{x}$ and $\mathbf{w}$ are reversibly restored from $\mathbf{y}$, which is defined as

$$
\begin{gathered}
x_{j}=y_{0}+\left\lfloor\frac{k-1}{n+1} \sum_{i=1}^{n}\left\lfloor\frac{y_{i}-y_{0}}{k}\right\rfloor+\frac{n(k-1)}{2(n+1)}\right\rfloor \\
+\left\lfloor\frac{y_{j}-y_{0}}{k}\right\rfloor, \\
w_{j-1}=y_{j}-k x_{j}+a_{n, k}(\mathbf{x}) \quad(j \neq 0) .
\end{gathered}
$$

\subsection{AGDE Method}

The generalized method in Sect. 2.1 was recently expanded into the AGDE method [19], which improves the visual quality by adaptively deciding on the $k$ according to the variance of each cover-block.

As outlined in Sect. 2.1, this method also embeds $\mathbf{w}$ in $\mathbf{x}$ to derive $\mathbf{y}$, from (1) with $k$. In general, grayscale images are presented in 8 -bit, i.e., $0 \leq x_{j} \leq 255$. $y_{j}$ should also be within $[0,255]$. To represent this range condition, sets $A$ and $A_{k}$ are introduced, as follows:

$$
\begin{aligned}
& A=\left\{\mathbf{x}=\left[x_{0}, x_{1}, \cdots, x_{n}\right] \mid 0 \leq x_{j} \leq 255\right\}, \\
& A_{k}=\left\{\mathbf{x} \in A \mid 0 \leq k x_{0}-a_{n, k}(\mathbf{x}) \leq 255,\right. \\
& \left.\quad 0 \leq k x_{j}-a_{n, k}(\mathbf{x}) \leq 256-k(j=1,2, \cdots, n)\right\} .
\end{aligned}
$$

Consequently, if $\mathbf{x}$ satisfies $\mathbf{x} \in A_{k}, \mathbf{y} \in A$ because of the definition of $\mathbf{y}$ in (1), and $\mathbf{y}$ has no-over/underflow.

In this method, $k$ of each cover-block is determined according to a given threshold parameter $T$ and the variance of the cover-block $V(\mathbf{x}) . \quad k$ is experimentally restricted as $\{1,2,4,8\}$ and determined as follows:

1. If $V(\mathbf{x}) \leq T / 49$ and $\mathbf{x} \in A_{8}$, escape algorithm with $k=8$.

2. If $V(\mathbf{x}) \leq T / 9$ and $\mathbf{x} \in A_{4}$, escape algorithm with $k=4$.

3. If $V(\mathbf{x}) \leq T$ and $\mathbf{x} \in A_{2}$, escape algorithm with $k=2$.

4. Else $k=1$.

This determination is shown in Fig. 2. Let $N$ and $k_{q}$ be 


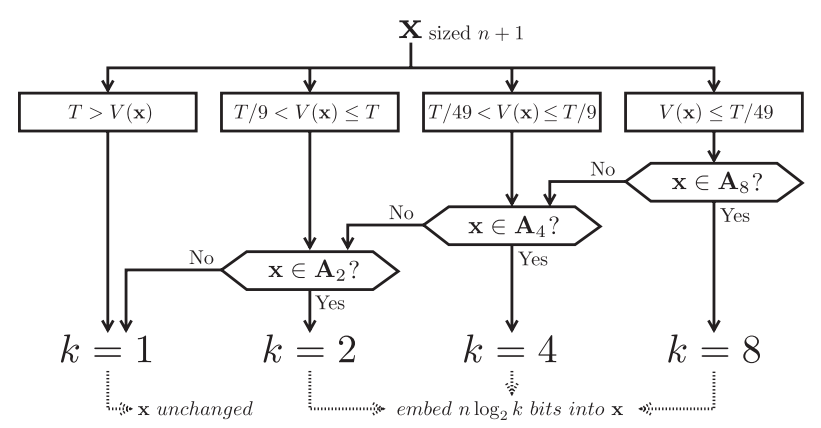

Fig. 2 Capacity parameter determination of the AGDE algorithm.

the cover-block number and $k$ of $q$-th cover-block $(q=$ $0,1 \cdots, N-1)$, respectively. The AGDE method embeds $n \sum_{q=0}^{N-1} \log _{2} k_{q}$ bits in $(n+1) N$ signals. Compared with the generalized method, the AGDE method achieves a higher peak signal-to-noise ratio (PSNR) between coverand stego-images, at the same capacity, because it adopts the optimal $k_{q}$ without over/underflow to minimize the overall distortions with a given capacity. Capacity parameters for each cover-block are regarded as side information required at extraction. We show how to embed them in the stego-image in Sect. 4.1.

\subsection{IntDCT}

Discrete cosine transform (DCT) is one of the most useful subband transform techniques and is applied for many signal processing applications, especially image/video coding standards. In the type-II DCT matrix $\mathbf{C}$ with a channel number $M$, its $(u, v)$ element $c_{u, v}(u, v=0,1, \cdots, M-1)$ is defined as

$$
c_{u, v}=\sqrt{\frac{2}{M}} \beta_{u} \cos \left(\frac{u(v+1 / 2) \pi}{M}\right),
$$

where $\beta_{u}$ is $1 / \sqrt{2}(u=0)$ or $1(u \neq 0)$.

IntDCT is an I2I-ST realized by the lifting factorization of DCT [21]-[24]. A $2 \times 2$ matrix can be factorized into the lifting structures as

$$
\left[\begin{array}{ll}
r_{0} & r_{1} \\
r_{2} & r_{3}
\end{array}\right]=\left[\begin{array}{cc}
1 & 0 \\
\frac{r_{3}-1}{r_{1}} & 1
\end{array}\right]\left[\begin{array}{cc}
1 & r_{1} \\
0 & 1
\end{array}\right]\left[\begin{array}{cc}
1 & 0 \\
\frac{r_{0}-1}{r_{1}} & 1
\end{array}\right],
$$

where $r_{0} r_{3}-r_{1} r_{2}=1$, and $r_{1} \neq 0$ [28]. Since the lifting structures with the rounding operations as shown in Fig. 3(a) achieve I2I-ST, original signals are reversibly restored via its inverse operations as shown in Fig. 3(b). In this paper, for simplicity, we utilize the 8-channel IntDCT in [21], which has least rounding operators and shows efficient image coding performance. The IntDCT is shown in Fig. 4, where $C_{q}^{p}=\cos (p \pi / q), S_{q}^{p}=\sin (p \pi / q)$, and the 4-channel lifting based Walsh-Hadamard transform is defined as

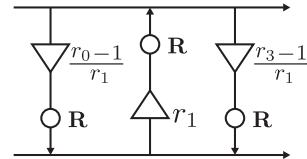

(a) Lifting factorization

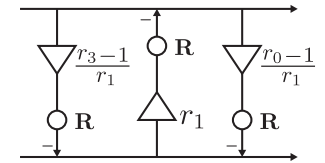

(b) Inverse
Fig. 3 Lifting structures of a $2 \times 2$ matrix, where $r_{0} r_{3}-r_{1} r_{2}=1$ and $r_{1} \neq 0$ ( $R$ means a rounding operator)

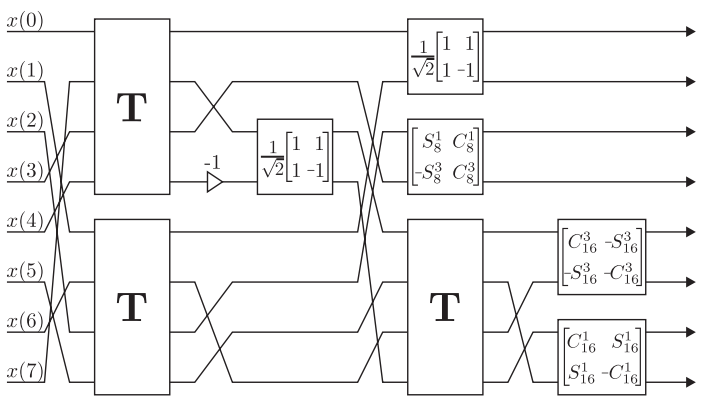

Fig. 4 8-channel IntDCT in [21].

$$
\begin{aligned}
\mathbf{T} & =\left[\begin{array}{llll}
0 & 0 & 1 & 0 \\
0 & 1 & 0 & 0 \\
1 & 0 & 0 & 0 \\
0 & 0 & 0 & 1
\end{array}\right]\left[\begin{array}{cccc}
1 & 0 & 0 & 0 \\
0 & 1 & 0 & 0 \\
0 & -1 & 1 & 0 \\
-1 & 0 & 0 & 1
\end{array}\right] \\
& \times\left[\begin{array}{cccc}
1 & 0 & 1 / 2 & 1 / 2 \\
0 & 1 & 1 / 2 & -1 / 2 \\
0 & 0 & 1 & 0 \\
0 & 0 & 0 & 1
\end{array}\right]\left[\begin{array}{cccc}
-1 & 0 & 0 & 0 \\
0 & -1 & 0 & 0 \\
1 & 0 & 1 & 0 \\
0 & -1 & 0 & 1
\end{array}\right] .
\end{aligned}
$$

The rotation matrices in Fig. 4 can be lifting-factorized as (6). Note that the frequency response of IntDCT is not strictly the same as the one of DCT due to the rounding errors.

\section{Adaptive Reversible Data Hiding via I2I-ST and AGDE Method}

\subsection{Formulation}

In this paper, we propose an adaptive reversible data hiding method by applying the AGDE method in Sect. 2.2 to the frequency domain obtained via the IntDCT in Sect. 2.3 as the I2I-ST method. This proposed method can easily be applied for any I2I-ST method with minor modifications.

Referring to Fig. 1, initially, we divide the cover-image into some cover-blocks with size $M \times(n+1)$. Experimentally, we set $M=8$ in this paper, but the number of $M$ can be easily adapted to an arbitrary number [23]. Let $\mathbf{X} \in \mathbb{Z}^{M \times(n+1)}$ be the cover-block, where $\mathbb{Z}^{M \times(n+1)}$ denotes a set of integer matrices with size $M \times(n+1)$.

Next, $\mathbf{X}$ is transformed by the IntDCT as $\mathbf{y}_{j}=\Phi\left(\mathbf{x}_{j}\right)$, where $\mathbf{x}_{j}$ and $\mathbf{y}_{j}$ are $j$-th column vectors of $\mathbf{X}$ and the transformed block $\mathbf{Y} \in \mathbb{Z}^{M \times(n+1)}$, respectively, and $\Phi$ means the function of the IntDCT operation in Fig. 4. In the proposed method, let $\mathbf{W} \in \mathbb{Z}^{M \times n}$ and $\mathbf{k} \in \mathbb{Z}^{M}$ be messages and capacity parameters of one cover-block. We embed $\mathbf{W}$ with $\mathbf{k}$ in 
$\mathbf{Y}$, and the produced stego-block in the frequency domain is denoted as $\mathbf{Z} \in \mathbb{Z}^{M \times(n+1)}$. This embedding is defined as

$$
z_{i, j}=\left\{\begin{array}{ll}
k_{i} y_{i, j}-a_{n, k_{i}}\left(\tilde{\mathbf{y}}_{i}\right) & \text { for } j=0 \\
k_{i} y_{i, j}-a_{n, k_{i}}\left(\tilde{\mathbf{y}}_{i}\right)+w_{i, j-1} & \text { for } j \neq 0
\end{array},\right.
$$

where $i=0,1, \cdots, M-1$, and $j=0,1, \cdots, n . y_{i, j}, z_{i, j}$, and $w_{i, j}$ are $(i, j)$ elements of $\mathbf{Y}, \mathbf{Z}$, and $\mathbf{W}, \tilde{\mathbf{y}}_{i}$ is $i$-th row vector of $\mathbf{Y}$, and $k_{i}$ is $i$-th element of $\mathbf{k}$. Needless to say, the range of $w_{i, j}$ is restricted by $k_{i}$. The subband transform is applied for each column vector of the cover-block. The embedding is applied for each row vector, i.e., each frequency subband in the frequency domain, because the variance of a region across some subbands is usually higher than one in only one subband. In particular, the variance of a region consisting of the direct current and other subbands critically increases. Therefore, we define the embedding of messages into each subband of $\mathbf{Y}$ as (8).

Finally, the stego-block in the space domain $\mathbf{S} \in$ $\mathbb{Z}^{M \times(n+1)}$ is derived from the inverse IntDCT of $\mathbf{Z}$. This process embeds $n \sum_{i=0}^{M-1} \log _{2} k_{i}$ bits into $M(n+1)$ pixels. It is the same embedding rate as the AGDE. At the receiver, the cover-block and messages are reversibly restored from the stego-block via the inverse process.

\subsection{Determination of Capacity Parameters}

In this section, we show the determination of the capacity parameters $\mathbf{k}$ at each cover-block. Initially, to avoid over/underflow, similar to sets $A$ and $A_{k}$, we define new sets $B$ and $B_{\mathbf{k}}$ as

$$
\begin{aligned}
B= & \left\{\mathbf{X} \in \mathbb{Z}^{M \times(n+1)} \mid 0 \leq x_{i, j} \leq 255\right\}, \\
B_{\mathbf{k}}= & \left\{\mathbf{X} \in B \mid 0-\sum_{p=0}^{M-1} c_{p, i}^{-}\left(k_{p}-1\right)+\alpha \leq \hat{e}_{i, j}\right. \\
& \left.\leq 255-\sum_{p=0}^{M-1} c_{p, i}^{+}\left(k_{p}-1\right)-\alpha\right\},
\end{aligned}
$$

where $\alpha$ is an arbitrary number as a margin and $c_{u, v}^{ \pm}$is $(u, v)$ element of $\mathbf{C}^{ \pm}$defined as

$$
c_{u, v}^{+}=\left\{\begin{array}{ll}
c_{u, v} & \text { if } c_{u, v} \geq 0 \\
0 & \text { if } c_{u, v}<0
\end{array}, \quad c_{u, v}^{-}=\left\{\begin{array}{ll}
0 & \text { if } c_{u, v} \geq 0 \\
c_{u, v} & \text { if } c_{u, v}<0
\end{array},\right.\right.
$$

and $c_{u, v}$ is $(u, v)$ element of the DCT matrix $\mathbf{C}$ in (5). $\hat{e}_{i, j}$ is defined with $y_{i, j}$ and $\tilde{\mathbf{y}}_{i}$ as

$$
\hat{e}_{i, j}=\sum_{p=0}^{M-1} c_{p, i}\left\{k_{p} y_{p, j}-a_{n, k_{p}}\left(\tilde{\mathbf{y}}_{p}\right)\right\} .
$$

Let $\hat{\mathbf{Z}} \in \mathbb{Z}^{M \times(n+1)}$ be the stego-block in the frequency domain with $\mathbf{W}=\mathbf{0}$ driven by (8), where $\mathbf{0}$ is a null matrix, i.e., its $(i, j)$ element $\hat{z}_{i, j}$ is defined as

$$
\hat{z}_{i, j}=k_{i} y_{i, j}-a_{n, k_{i}}\left(\tilde{\mathbf{y}}_{i}\right),
$$

and $z_{i, j}$ and $\hat{z}_{i, j}$ are related as $z_{i, j}=\hat{z}_{i, j}+w_{i, j-1}(j \neq 0)$. Let $\hat{\mathbf{E}} \in \mathbb{R}^{M \times(n+1)}$ be the stego-block in the space domain derived from the inverse DCT of $\hat{\mathbf{Z}}$ as $\hat{\mathbf{E}}=\mathbf{C}^{T} \hat{\mathbf{Z}}$. Its $(i, j)$ element $\hat{e}_{i, j}$ is then defined as (11). Similarly, let $\mathbf{E}$ be $\mathbf{E}=\mathbf{C}^{T} \mathbf{Z}$. The relation of $\mathbf{E}$ and $\hat{\mathbf{E}}$ is represented as

$$
e_{i, j}=\left\{\begin{array}{ll}
\hat{e}_{i, j} & \text { for } j=0 \\
\hat{e}_{i, j}+\sum_{p=0}^{M-1} c_{p, i} w_{p, j-1} & \text { for } j \neq 0
\end{array},\right.
$$

where $e_{i, j}$ is $(i, j)$ element of $\mathbf{E}$. From the definition of $\mathbf{W}, w_{i, j}$ is restricted in $\left[0, k_{i}-1\right]$. Hence, the interval of the second term of (13) for $j \neq 0$ is derived as $\left[\sum_{p=0}^{M-1} c_{p, i}^{-}\left(k_{p}-1\right), \sum_{p=0}^{M-1} c_{p, i}^{+}\left(k_{p}-1\right)\right] . \quad$ Therefore, if $0-$ $\sum_{p=0}^{M-1} c_{p, i}^{-}\left(k_{p}-1\right) \leq \hat{e}_{i, j}$ in $B_{\mathbf{k}}, e_{i, j}$ satisfies $0 \leq e_{i, j}$ with arbitrary messages, i.e., the pixel avoids an underflow. The overflow verification is defined in the same way. Finally, similar to the case of $A$ and $A_{k}$, if $X \in B_{\mathbf{k}}$, there is no-over/underflow in the corresponding $\mathbf{S}$ and $\mathbf{E}$. However, for $\mathbf{S}$, the condition does not exactly guarantee no-over/underflow, as discussed later.

By using the subset $B_{\mathbf{k}}$ and a given threshold parameter $T$, the determination algorithm of $\mathbf{k}$ shown in Fig. 5 is defined as follows:

1. The initial $\mathbf{k}$ is determined as

$$
k_{i}=\left\{\begin{array}{ll}
1 & \text { for } V\left(\tilde{\mathbf{y}}_{i}\right)>T \\
2 & \text { for } T / 9<V\left(\tilde{\mathbf{y}}_{i}\right) \leq T \\
4 & \text { for } T / 49<V\left(\tilde{\mathbf{y}}_{i}\right) \leq T / 9 \\
8 & \text { for } V\left(\tilde{\mathbf{y}}_{i}\right) \leq T / 49
\end{array},\right.
$$

where $V\left(\tilde{\mathbf{y}}_{i}\right)$ is a variance of $\tilde{\mathbf{y}}_{i}$

2. If $\mathbf{X} \in B_{\mathbf{k}}$, escape algorithm with $\mathbf{k}$.

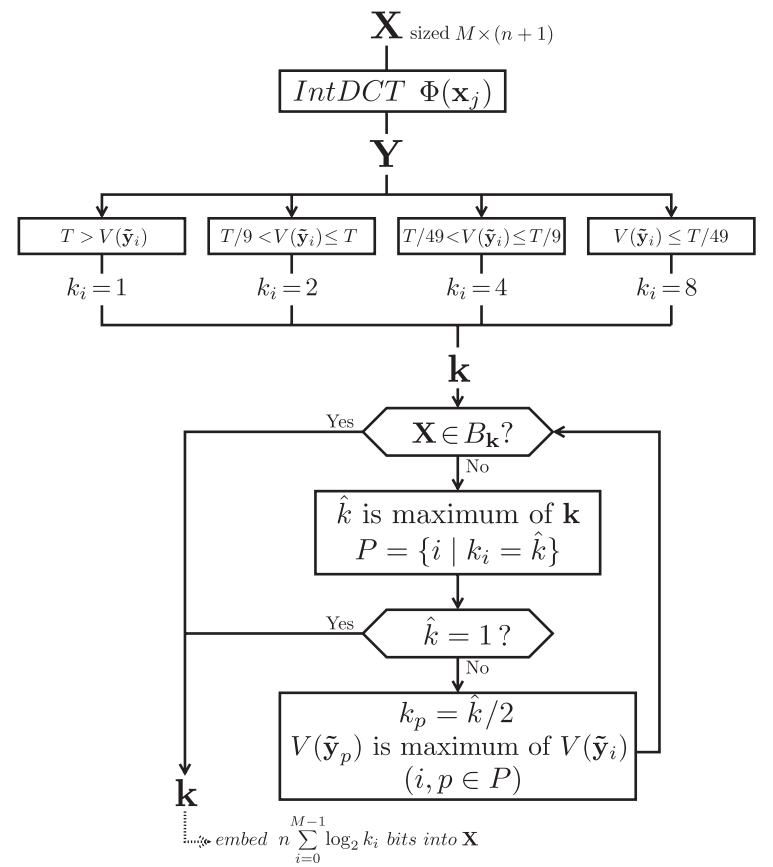

Fig. 5 Proposed determination of capacity parameters. 
3. Let $\hat{k}$ and $P$ be a maximum of $\mathbf{k}$ and a set $\left\{i \mid k_{i}=\hat{k}\right\}$, respectively.

4. If $\hat{k}=1$, escape algorithm with $\mathbf{k}$.

5. $k_{p}=\hat{k} / 2$, where $p(p \in P)$ is an index whose $V\left(\tilde{\mathbf{y}}_{p}\right)$ is the maximum of $V\left(\tilde{\mathbf{y}}_{i}\right)$. Go to Step 2.

As mentioned above, the condition $\mathbf{X} \in B_{\mathbf{k}}$ does not exactly guarantee that the corresponding $\mathbf{S}$ has noover/underflow pixels. The rounding errors in each lifting step cannot be exactly predicted due to depending on input signals. If the optimal $\mathbf{k}$ is required, over/underflow is checked after $\mathbf{S}$ is calculated with arbitrary $\mathbf{k}$, iteratively. However, in this case, the determination should wait until the previous blocks are finished, i.e., in a non-parallel process. This is not useful because it requires a high computational cost and takes a long time to process. Therefore, the complexity is reduced by introducing the margin $\alpha$ as defined in (9).

\section{Implementation Structure}

This section briefly presents an implementation algorithm [19].

\subsection{Embedding Algorithm}

We focus on how to treat the capacity parameters of each block. A set of the capacity parameters is called a location map (LM). The embedding algorithm shown in Fig. 6 is implemented as follows:

1. The cover-image is divided into some cover-blocks that are indexed along the predetermined order.

2. $\mathbf{k}$ of each cover-block is determined and the LM is constructed as a sequence of $\mathbf{k}$.

3. The cover-blocks are classified into two categories $X_{1}$ and $X_{2}$ according to the length of the encoded LM.

4. A part of the messages is embedded into $X_{1}$ according to the LM, and the stego-blocks in the space domain are set to $S_{1}$.

5. The LSBs of $S_{1}$ are replaced with the encoded LM and then encoded.
6. The encoded LSBs and the residual messages are embedded into $X_{2}$ and the stego-blocks are set to $S_{2}$.

7. The stego-image is constructed from $S_{1}$ and $S_{2}$.

The details of the classification in Step 3 are shown here. Let $N$ and $L$ be the block number and length of the encoded LM, respectively $L$ is stored as the header of the encoded LM. Since $L$ is up to $2 M N$, a length of the header $L_{h}$ is $\left\lceil\log _{2}(2 M N-1)\right\rceil$, where $\lceil *\rceil$ is the ceiling function. Hence, to store the header and the encoded LM via the LSB replacement, $X_{1}$ should be a set of the former $\left\lceil\left(L+L_{h}\right) / M(n+1)\right\rceil$ blocks. Of course, $X_{2}$ is a set of the residual latter blocks.

For the simulation discussed later in Sect. 5, the conditions are as follows: We used a raster scan as the predetermined order. The LM is encoded by Huffman coding and arithmetic coding and the replaced LSBs are encoded by arithmetic coding [29], if necessary. In the extraction, we can divide the stego-image into $S_{1}$ and $S_{2}$ via reading the first $L_{h}$ LSBs of the stego-image along the predetermined order.

If the stego-image should be locked, the above embedding procedure is not needed, and the stego-image is simply derived from the proposed method. In this case, the LM is recognized as the key.

\subsection{Extraction Algorithm}

The extraction procedure corresponding to the algorithm in Sect. 4.1 is as follows:

1. By reading the LSBs, the stego-image is divided into $S_{1}$ and $S_{2}$ and the encoded LM is extracted.

2. From $S_{2}, X_{2}$ is restored, and the replaced LSBs and residual messages are extracted based on the LM.

3. The LSBs of $S_{1}$ are restored from the replaced LSBs, $X_{1}$ is restored, and the messages are extracted.

4. The cover-image is restored from $X_{1}$ and $X_{2}$.

\section{Simulation}

We performed simulations to compare the proposed method

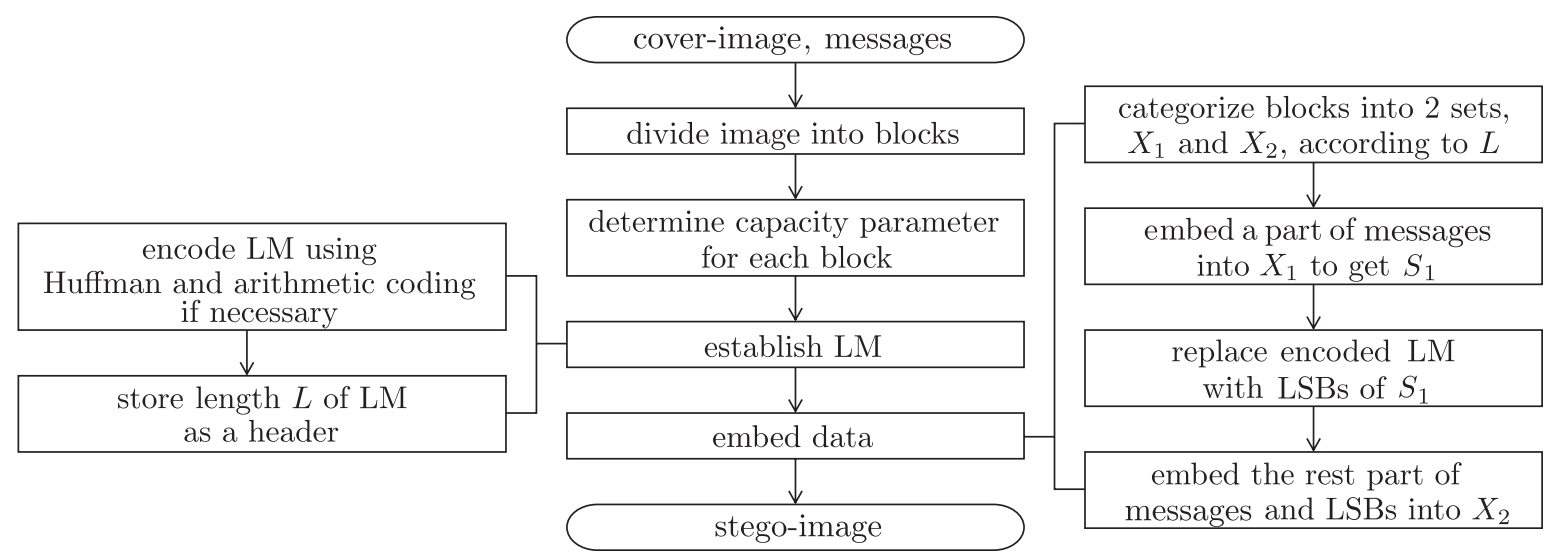

Fig. 6 Flowchart of data embedding. 


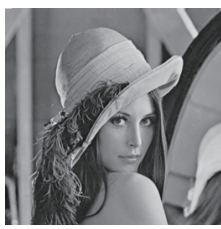

(a) Lena

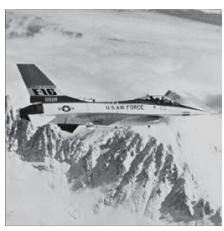

(b) Airplane

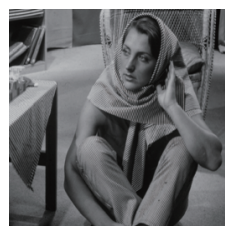

(c) Barbara

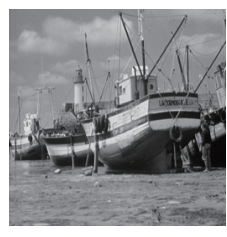

(d) Boat

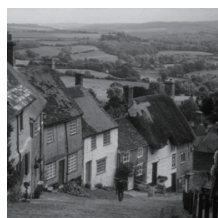

(e) Goldhill

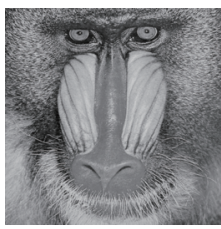

(f) Baboon

Fig. 7 Test images.
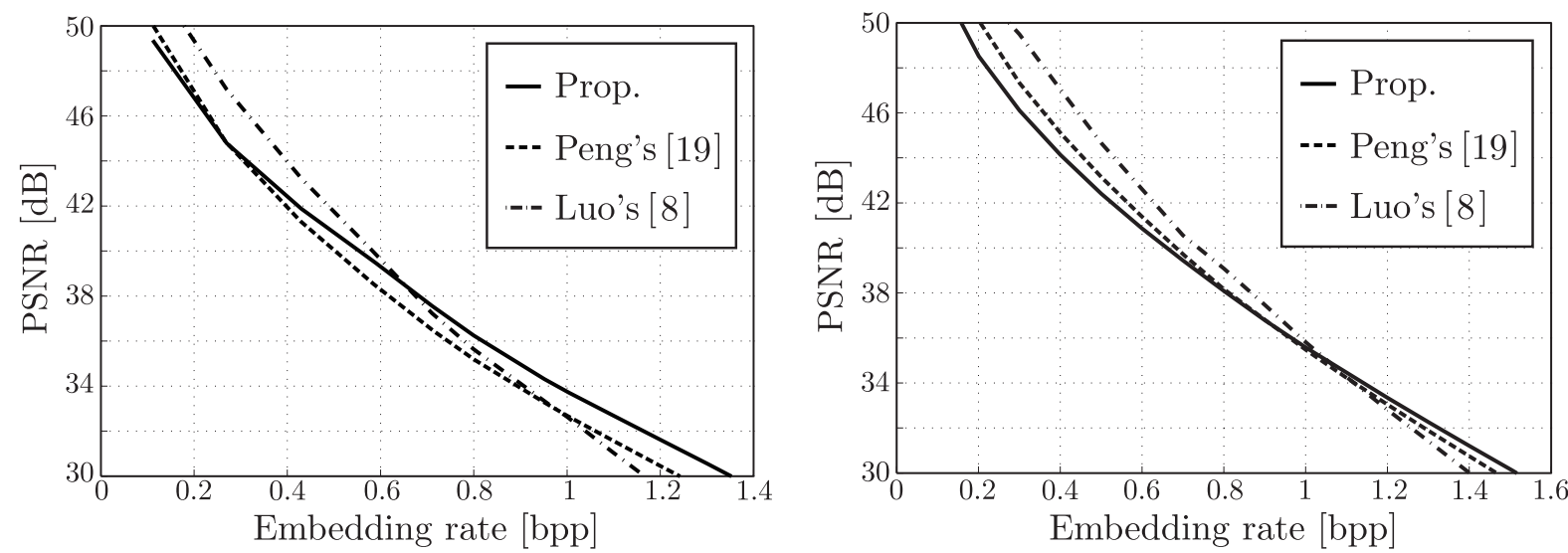

(a) Lena

(b) Airplane
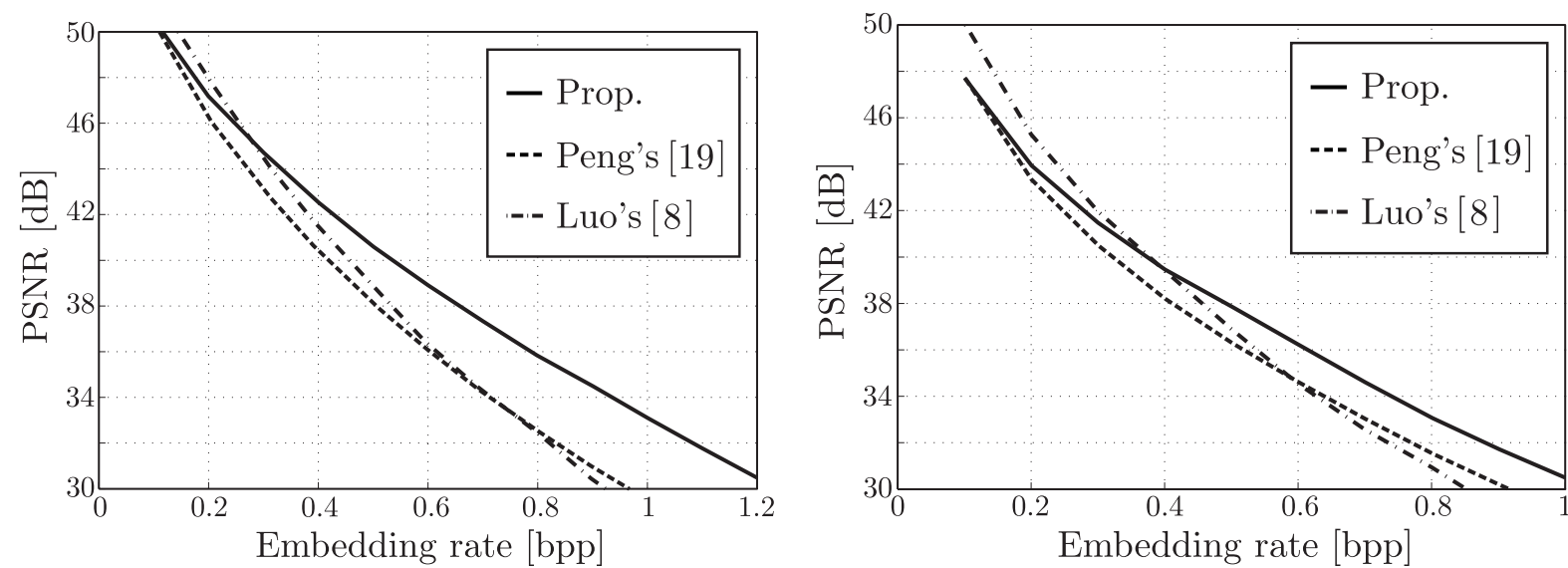

(c) Barbara

(d) Boat

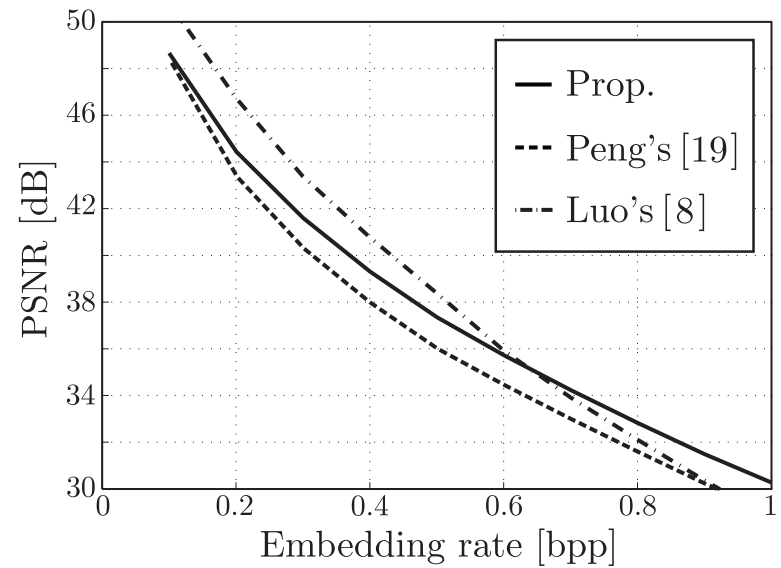

(e) Goldhill

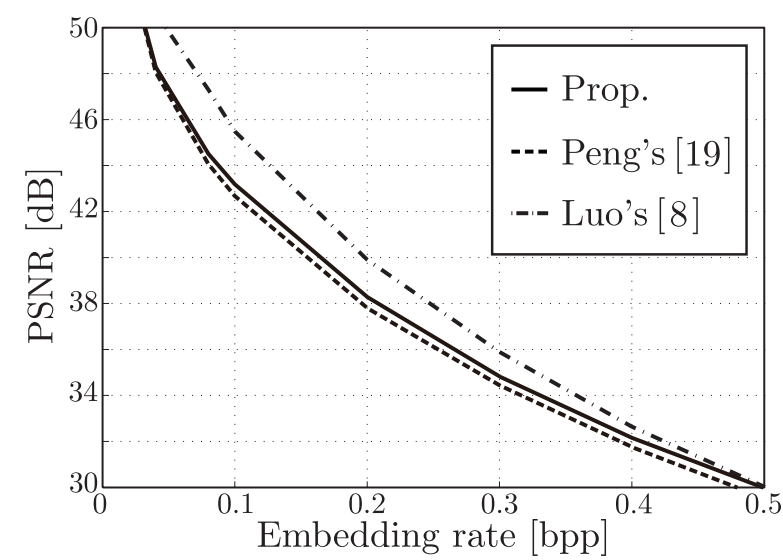

(f) Baboon

Fig. 8 Embedding RD curves. 
Table 1 Embedding rates [bpp], when PSNR is $30 \mathrm{~dB}$.

\begin{tabular}{c||c|c|c}
\hline Image & Luo's [8] & Peng's [19] & Prop. \\
\hline Lena & 1.16 & 1.23 & $\mathbf{1 . 3 4}$ \\
\hline Airplane & 1.39 & 1.46 & $\mathbf{1 . 5 0}$ \\
\hline Barbara & 0.90 & 0.96 & $\mathbf{1 . 2 3}$ \\
\hline Boat & 0.84 & 0.91 & $\mathbf{1 . 0 5}$ \\
\hline Goldhill & 0.91 & 0.92 & $\mathbf{1 . 0 3}$ \\
\hline Baboon & $\mathbf{0 . 5 0}$ & 0.47 & $\mathbf{0 . 5 0}$ \\
\hline
\end{tabular}

Table 2 PSNRs of stego-images [dB].

\begin{tabular}{c|c||c|c|c}
\hline Image & Rate & Luo's [8] & Peng's [19] & Prop. \\
\hline & 0.8 & 35.63 & 35.18 & $\mathbf{3 6 . 2 4}$ \\
Lena & 1.0 & 32.63 & 32.67 & $\mathbf{3 3 . 7 0}$ \\
& 1.2 & 29.47 & 30.44 & $\mathbf{3 1 . 6 0}$ \\
\hline \hline \multirow{3}{*}{ Airplane } & 0.8 & $\mathbf{3 9 . 0 8}$ & 38.17 & 38.07 \\
& 1.0 & $\mathbf{3 5 . 8 1}$ & 35.41 & 35.58 \\
& 1.2 & 32.81 & 32.99 & $\mathbf{3 3 . 3 1}$ \\
\hline \hline & 0.8 & 32.37 & 32.48 & $\mathbf{3 5 . 8 4}$ \\
Barbara & 1.0 & 28.67 & 29.52 & $\mathbf{3 3 . 0 8}$ \\
& 1.2 & 24.43 & 26.77 & $\mathbf{3 0 . 4 6}$ \\
\hline \hline & 0.8 & 30.95 & 31.53 & $\mathbf{3 3 . 0 6}$ \\
Boat & 1.0 & 27.51 & 28.94 & $\mathbf{3 0 . 4 7}$ \\
& 1.2 & 24.17 & 26.60 & $\mathbf{2 8 . 2 1}$ \\
\hline \hline & 0.8 & 32.11 & 31.58 & $\mathbf{3 2 . 8 3}$ \\
Goldhill & 1.0 & 28.75 & 28.96 & $\mathbf{3 0 . 2 6}$ \\
& 1.2 & 25.32 & 26.61 & $\mathbf{2 7 . 8 8}$ \\
\hline \hline & 0.8 & 23.62 & 24.68 & $\mathbf{2 5 . 0 5}$ \\
Baboon & 1.0 & 19.56 & 22.26 & $\mathbf{2 2 . 4 6}$ \\
& 1.2 & - & - & - \\
\hline
\end{tabular}

with Luo's and Peng's methods [8], [19], which are state-ofthe-art methods of reversible data hiding, using the MATLAB programming language. According to [19], the block size of Peng's method is $4 \times 4$, so $n=15$ in both Peng's and the proposed methods. We set $\alpha=0$ in (9) and experimentally determined the threshold parameter $T$ so that it was a large enough integer number to achieve the desired capacity. The three methods were applied to six standard test images $(512 \times 512,8$-bit grayscale), shown in Fig. 7. A random bit string was used as the message.

Figure 8 shows the embedding rate-distortion (RD) curves. The visual qualities of the stego-images are objectively measured using PSNR. The embedding rate is presented in bpp. Table 1 shows the embedding rates when the PSNRs are $30 \mathrm{~dB}$. From these figures and the table, it is clear that the proposed method achieves the highest embedding capacity. Moreover, the proposed method has a better visual quality at high embedding rates. Unfortunately, at low embedding rates, Luo's HS method delivers a better visual quality because the proposed method embeds not only messages but also the LM. In fact, the encoded LM seriously affects the embedding rates, e.g., the encoded LM with $15 \times 10^{3}$ bits at $0.2 \mathrm{bpp}$ extends to $23 \%$ in overall capacity.

Table 2 objectively shows the visual qualities at high embedding rates. At $1.2 \mathrm{bpp}$ in Baboon, none of the methods are able to embed. The proposed method shows better results than the conventional ones, especially for images
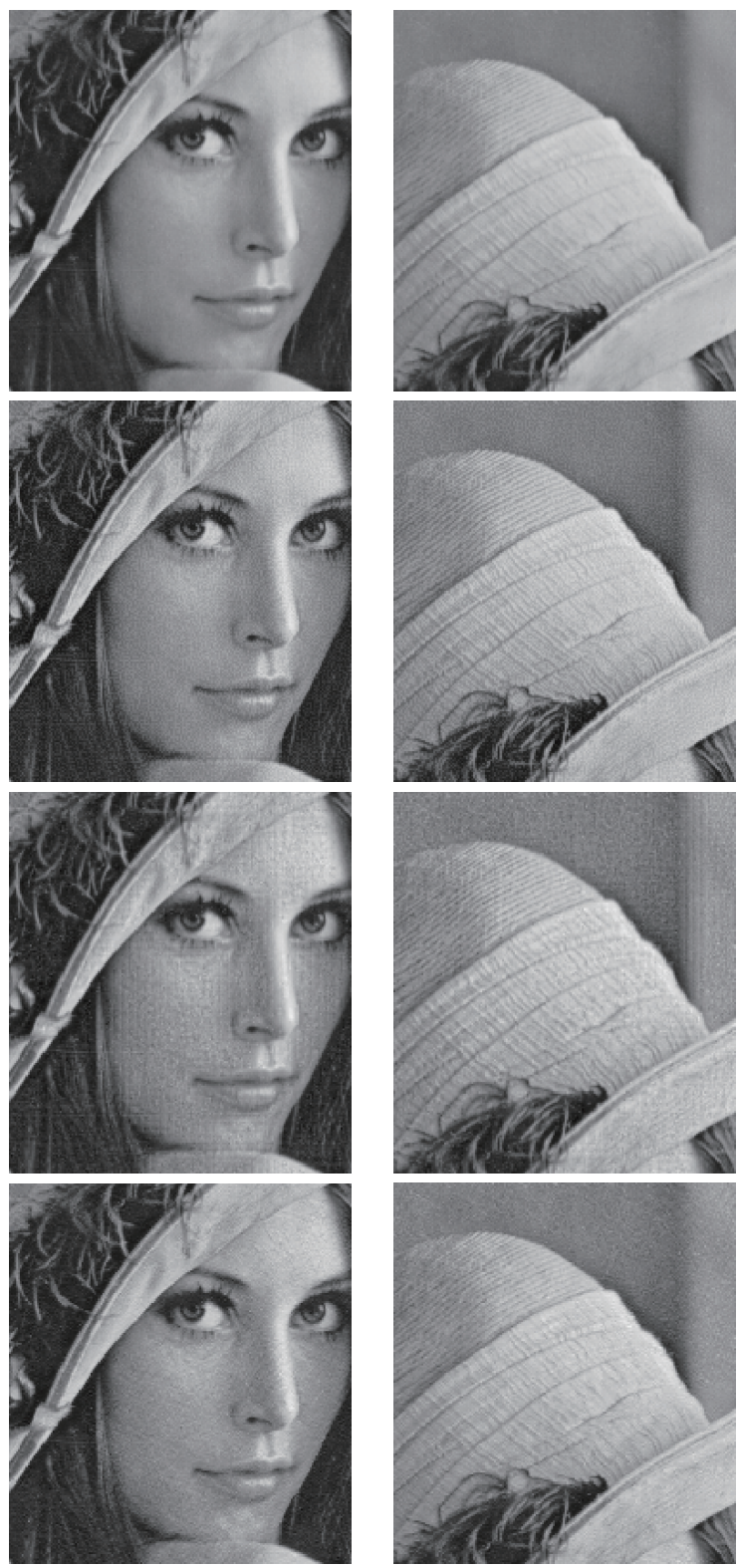

Fig. 9 Particular areas of stego-images of Lena at 1.0 bpp: (1st row) Cover-image (2nd row) Luo's [8], (3rd row) Peng's [19], (4th row) Prop.

with rich high frequency components such as Barbara. On the other hand, in the case of very smooth images such as Airplane, the proposed method shows results comparable with Luo's. The rate range, in which the proposed method has the best result, is wide if the image has rich high frequency components, and vice versa. However, the DE methods are ineffective for extremely textured images such as Baboon. Figure 9 shows specific areas of the stego-images of Lena at $1.0 \mathrm{bpp}$. Although the particular distortions in Peng's method are perceived, the distortions in the proposed images are not easily noticeable because they are similar to ordinary random noise. This is because the distortions produced by embedding in the frequency domain are randomly spread to the neighborhood pixels via the inverse frequency 
transform with oscillated atoms of high frequency subbands. Also, the proposed method does not produce an excessive contrast enhancement, as Luo's method does. According to the visual policy of data hiding that the modification of embedding should not be perceptually recognized, the proposed method has better perceptual results because the particular distortion has a higher risk of recognizing the hiding than a random noise. Consequently, our method objectively and perceptually shows better visual quality than the conventional methods at the same embedding rate.

\section{Conclusion}

In this paper, we proposed an adaptive reversible data hiding method in the frequency domain obtained via integer-tointeger subband transform (I2I-ST) and the adaptive generalized difference expansion (AGDE) method. Natural images in the frequency domain usually have a lower variance. In addition, the distortions generated by embedding are distributed to neighborhoods via the inverse subband transform. Therefore, the AGDE method in the frequency domain obtained by an I2I-FT is more suitable for reversible data hiding than one in the space domain. As a result, the proposed method objectively and perceptually showed an excellent performance. For even better visual quality, we should apply other I2I-FTs and adaptive threshold parameters for each subband.

\section{Acknowledgments}

This work was supported by Grant-in-Aid for JSPS Fellows, and JSPS KAKENHI Grant Number 25820152.

\section{References}

[1] C.H. Lee and Y.K. Lee, "An adaptive digital image watermarking technique for copyright protection," IEEE Trans. Consum. Electron., vol.45, no.4, pp.1005-1015, Nov. 1999.

[2] C.W. Tang and H.M. Hang, "A feature-based robust digital image watermarking scheme," IEEE Trans. Signal Process., vol.51, no.4, pp.950-959, April 2003.

[3] F.A.P. Petitcolas, R.J. Anderson, and M.G. Kuhn, "Information hiding-A survey," Proc. IEEE, vol.87, no.7, pp.1062-1078, July 1999

[4] Z. Ni, Y.Q. Shi, N. Ansari, and W. Su, "Reversible data hiding," IEEE Trans. Circuits Syst. Video Technol., vol.16, no.3, pp.354362, March 2006.

[5] P. Tsai, Y.C. Hu, and H.L. Yeh, "Reversible image hiding scheme using predictive coding and histogram shifting," Signal Process., vol.89, pp.1129-1143, June 2009.

[6] W.L. Tai, C.M. Yeh, and C.C. Chang, "Reversible data hiding based on histogram modification of pixel differences," IEEE Trans. Circuits Syst. Video Technol., vol.19, no.6, pp.906-910, June 2009.

[7] W. Hong, T.S. Chen, Y.P. Chang, and C.W. Shiu, "A high capacity reversible data hiding scheme using orthogonal projection and prediction error modification," Signal Process., vol.90, no.11, pp.29112922, Nov. 2010.

[8] L. Luo, Z. Chen, M. Chen, X. Zeng, and Z. Xiong, "Reversible image watermarking using interpolation technique," IEEE Trans. Inf. Forensics Security, vol.5, no.1, pp.187-193, March 2010.
[9] C.C. Chen and D.S. Kao, "DCT-based reversible image watermarking approach," Proc. Int. Conf. Intell. Inf. Hiding Multimedia Signal Process., pp.489-492, Nov. 2007.

[10] S. Lee, C.D. Yoo, and T. Kalker, "Reversible image watermarking based on integer-to-integer wavelet transform," IEEE Trans. Inf. Forensics Security, vol.2, no.3, pp.321-330, Sept. 2007.

[11] C.C. Lin and P.F. Shiu, "DCT-based reversible data hiding scheme," J. Software, vol.5, no.2, pp.214-224, Feb. 2010.

[12] J. Tian, "Reversible data embedding using a difference expansion," IEEE Trans. Circuits Syst. Video Technol., vol.13, no.8, pp.890896, Aug. 2003.

[13] D.M. Thodi and J.J. Rodriguez, "Expansion embedding techniques for reversible watermarking," IEEE Trans. Image Process., vol.16, no.3, pp.721-730, March 2007.

[14] H.L. Jin, M. Fujiyoshi, and H. Kiya, "Lossless data hiding in the spatial domain for high quality images," IEICE Trans. Fundamentals, vol.E90-A, no.4, pp.771-777, April 2007.

[15] Y. Hu, H.K. Lee, and J. Li, "DE-based reversible data hiding with improved overflow location map," IEEE Trans. Circuits Syst. Video Technol., vol.19, no.2, pp.250-260, Feb. 2009.

[16] A.M. Alattar, "Reversible watermark using the difference expansion of a generalized integer transform," IEEE Trans. Image Process., vol.13, no.8, pp.1147-1156, Aug. 2004.

[17] S. Weng, Y. Zhao, J.S. Pan, and R. Ni, "Reversible watermarking based on invariability and adjustment on pixel pairs," IEEE Signal Process. Lett., vol.15, pp.721-724, 2008.

[18] C. Wang, X. Li, and B. Yang, "High capacity reversible image watermarking based on integer transform," Proc. Int. Conf. Image Process., pp.217-220, Sept. 2010.

[19] F. Peng, X. Li, and B. Yang, "Adaptive reversible data hiding scheme based on integer transform," Signal Process., vol.92, no.1, pp.54-62, Jan. 2012.

[20] ISO/IEC 10918-1, Information technology — Digital compression and coding of continuous-tone still images Requirements and Guidelines.

[21] S. Fukuma, K. Ohyama, M. Iwahashi, and N. Kambayashi, "Lossless 8-point fast discrete cosine transform using lossless hadamard transform," IEICE Technical Report, Oct. 1999.

[22] S. Chokchaitam, M. Iwahashi, and S. Jitapunkul, "A new unified lossless/lossy image compression based on a new integer DCT," IEICE Trans. Inf. \& Syst., vol.E88-D, no.2, pp.403-413, Feb. 2005.

[23] T. Suzuki and M. Ikehara, " $M$-channel integer discrete cosine transform using block lifting factorization," IEICE Trans. Fundamentals (Japanese Edition), vol.J92-A, no.10, pp.659-668, Oct. 2009.

[24] T. Suzuki and M. Ikehara, "Integer DCT based on direct-lifting of DCT-IDCT for lossless-to-lossy image coding," IEEE Trans. Image Process., vol.19, no.11, pp.2958-2965, Nov. 2010.

[25] Y.J. Chen and K.S. Amaratunga, " $M$-channel lifting factorization of perfect reconstruction filter banks and reversible $M$-band wavelet transforms," IEEE Trans. Circuits Syst. II, Analog Digit. Signal Process., vol.50, no.12, pp.963-976, Dec. 2003.

[26] T. Suzuki, Y. Tanaka, and M. Ikehara, "Lifting-based paraunitary filterbanks for lossy/lossless image coding," IEICE Trans. Fundamentals (Japanese Edition), vol.J89-A, no.11, pp.950-959, Nov. 2006.

[27] T. Suzuki, M. Ikehara, and T.Q. Nguyen, "Generalized block-lifting factorization of $M$-channel biorthogonal filter banks for lossy-tolossless image coding," IEEE Trans. Image Process., vol.21, no.7, pp.3220-3228, July 2012.

[28] F.A.M.L. Bruekers and A.W.M. van den Enden, "New networks for perfect inversion and perfect reconstruction," IEEE J. Sel. Areas Commun., vol.10, no.1, pp.129-137, Jan. 1992.

[29] R.C. Gonzalez and R.E. Woods, Digital Image Processing, Prentice Hall, 2002. 


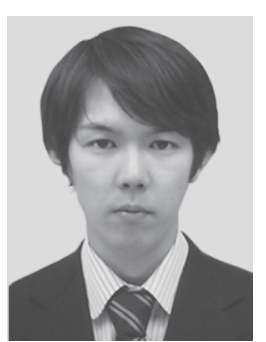

Taichi Yoshida received the B.E., M.E., and Ph.D. degrees in electrical engineering from Keio University, Yokohama, Japan, in 2006, 2008, and 2013, respectively. He is currently a Ph.D. at Keio University, Yokohama, Japan, under the supervision of Prof. Masaaki Ikehara, and a Research Fellow of the JSPS. His research interests are in the field of filter bank design and its image coding application.

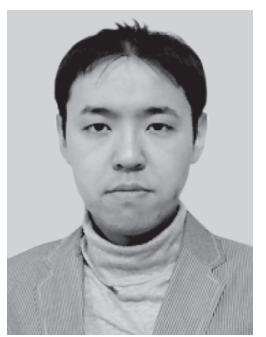

Taizo Suzuki received the B.E., M.E. and $\mathrm{Ph} . \mathrm{D}$. degrees in electrical engineering from Keio University, Yokohama, Japan, in 2004, 2006 and 2010, respectively. He joined Toppan Printing Co., Ltd., Tokyo, Japan, from 2006 to 2008. From 2008 to 2011, he was a Research Associate of Global COE (C-12). From 2010 to 2011, a Research Fellow of the JSPS and a Visiting Scholar at Video Processing Group, the University of California, San Diego, La Jolla, CA, respectively. From 2011 to 2012, he was an Assistant Professor in the Department of Electrical and Electronic Engineering, College of Engineering, Nihon University, Koriyama, Japan. Since 2012, he is an Assistant Professor in the Faculty of Engineering, Information and Systems, University of Tsukuba, Tsukuba, Japan. His current research interests are filter bank theory/design and its application for image/video.

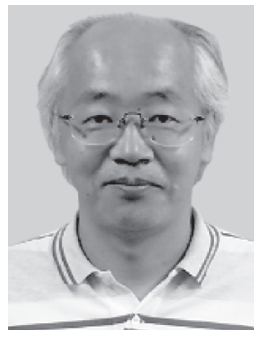

Masaaki Ikehara received the B.E., M.E. and Dr.Eng.degrees in electrical engineering from Keio University, Yokohama, Japan, in 1984, 1986, and 1989, respectively. He was Appointed Lecturer at Nagasaki University, Nagasaki, Japan, from 1989 to 1992. In 1992, he joined the Faculty of Engineering, Keio University. From 1996 to 1998, he was a visiting researcher at the University of Wisconsin, Madison, and Boston University, Boston, MA. He is currently a Full Professor with the Department of Electronics and Electrical Engineering, Keio University. His research interests are in the areas of multirate signal processing, wavelet image coding, and filter design problems. 